\title{
Intraperitoneal paclitaxel: pharmacology, clinical results and future prospects
}

\author{
Paul H. Sugarbaker \\ Center for Gastrointestinal Malignancies, MedStar Washington Hospital Center, Washington, DC, USA \\ Correspondence to: Paul H. Sugarbaker, MD, FACS, FRCS. 106 Irving St., NW, Suite 3900, Washington, DC 20010 , USA. \\ Email: Paul.Sugarbaker@outlook.com.
}

\begin{abstract}
Paclitaxel administered into the peritoneal cavity is a chemotherapy agent that shows unusually prolonged retention within the peritoneal space. Using this pharmacokinetic fact as a starting point, the use of this drug to benefit patients with peritoneal metastases was investigated. The pharmacokinetics and drug characteristics of paclitaxel were identified from the oncologic literature. The experience to date with ovarian cancer, malignant peritoneal mesothelioma, gastric cancer and pancreas cancer was explored. Paclitaxel given by repeated instillation through an intraperitoneal port has demonstrable responses in ovarian cancer, peritoneal mesothelioma, gastric cancer and pancreas cancer when peritoneal metastases are present. Its role for prevention of peritoneal metastases in patients at high risk seems less well established. Randomized controlled studies have been positive in ovarian cancer but not in other diseases with peritoneal dissemination. A randomized controlled study in gastric cancer with peritoneal metastases produced suggestive but not conclusive results. Conversion surgery after repeated treatments with intraperitoneal paclitaxel has been reported with gastric cancer and pancreas cancer with peritoneal metastases. The pharmacology of intraperitoneal paclitaxel strongly suggest that intraperitoneal administration should be of benefit to prevent or treat peritoneal metastases. Protocols that the oncologist can follow to realize these potential benefits are not as yet available.
\end{abstract}

Keywords: Hyperthermic intraperitoneal chemotherapy (HIPEC); early postoperative intraperitoneal chemotherapy (EPIC); normothermic intraperitoneal chemotherapy (NIPEC); NIPS; neoadjuvant chemotherapy; ovarian cancer; gastrointestinal cancer; intraperitoneal port

Submitted May 22, 2020. Accepted for publication Jun 04, 2020.

doi: 10.21037/jgo-2020-03

View this article at: http://dx.doi.org/10.21037/jgo-2020-03

\section{Introduction}

Documentation of the remarkable retention of paclitaxel within the peritoneal space after intraperitoneal administration in a large volume of intraperitoneal fluid was first published by Markman et al. in a Gynecologic Oncology Group phase I trial (1). Twenty-five patients were administered escalating doses of intraperitoneal paclitaxel and a tolerable dose on a monthly administration of $125 \mathrm{mg} / \mathrm{m}^{2}$ was observed. The most interesting and important observation following intraperitoneal administration was the marked difference of intraperitoneal paclitaxel concentration to intravenous concentration.
The area under the curve ratio (AUC) of intraperitoneal concentration over time compared to systemic compartment concentration over time was $1,000: 1$. Systemic concentrations of paclitaxel were quite variable but showed peak plasma levels associated with a major biologic effect (2). A major role for intraperitoneal paclitaxel in ovarian cancer was suggested.

Surprisingly, when the Gynecologic Oncology Group implemented its second trial of intraperitoneal chemotherapy for ovarian cancer the paclitaxel was administered systemically in both arms of the trial. Intravenous $v s$. intraperitoneal cisplatin was the clinical science question that was addressed. The trial showed 
marginal statistical significance. The possible large benefits of intraperitoneal paclitaxel in ovarian cancer were not studied (3).

Working with a knowledge of the large pharmacologic advantages of intraperitoneal paclitaxel, the potential uses of this drug by a regional delivery system is reviewed in this manuscript. A description of the pharmacokinetics of intraperitoneal paclitaxel is the starting point for our efforts. Its use in ovarian cancer, malignant peritoneal mesothelioma, gastric cancer, and pancreas cancer will be investigated.

\section{Pharmacokinetics of intraperitoneal paclitaxel}

Mohamed and coworkers reviewed the experience at the Washington Cancer Institute with the pharmacokinetics of intraperitoneal paclitaxel in patients with peritoneal metastases and in patients with malignant peritoneal

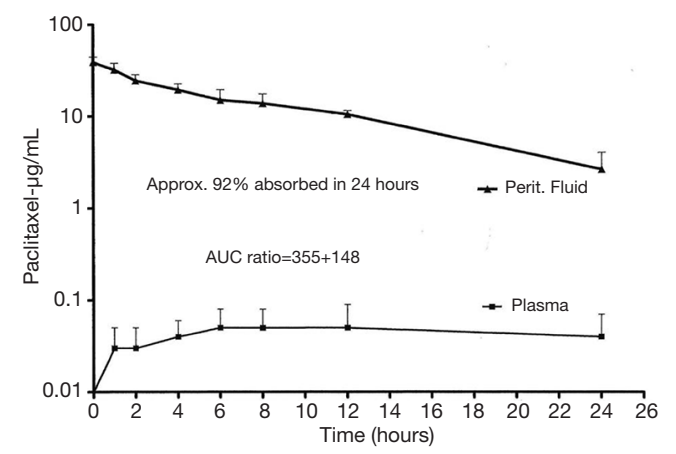

Figure 1 Pharmacokinetic study of normothermic intraperitoneal chemotherapy (NIPEC) with paclitaxel in patients with diffuse malignant peritoneal mesothelioma. Dose of paclitaxel was $20 \mathrm{mg} / \mathrm{m}^{2}$. Reprinted from (5) with permission. mesothelioma (4). The drug was dissolved in 1 liter of $1.5 \%$ dextrose peritoneal dialysis solution and instilled as rapidly as possible following a surgical procedure that divided all peritoneal adhesions. The access of the chemotherapy solution to all parietal and visceral peritoneal surfaces should be optimal in these patients immediately postcytoreduction. There was a large variation of the AUC ratio. It was $355 \pm 148$ (5). This AUC ratio is not as high as that reported by Markman of 1,000 (1). The clearance from the peritoneal space in the Washington Cancer Institute data was predicted to be more rapid because of wider distribution of the chemotherapy solution as a result of a total absence of adhesions within the abdomen and pelvis (Figure 1). At the end of 24 hours, $92 \%$ of the drug had cleared the peritoneal space suggesting that repeated daily doses in the early postoperative period would be efficacious.

Mohamed and coworkers showed convincingly that the solution that contains the paclitaxel is important in the prolongation of direct exposure of peritoneal metastases and cancer chemotherapy. They compared the pharmacokinetics of intraperitoneal paclitaxel in an aqueous solution to the drug in a starch solution (6\% hydroxyethyl starch). Because of its large molecular size, starch particles cause a prolonged retention of fluid within the peritoneal space. The concentration of paclitaxel was unchanged by the starch solution as compared to the aqueous solution. However, a significant difference in volume of chemotherapy solution was demonstrated. Figure 2 shows the significant total volume difference of intraperitoneal fluid with hetastarch carrier solution $v s$. an aqueous carrier solution $(\mathrm{P}=0.0022)$. Also, a larger total volume of hetastarch carrier solution indicates a greater peritoneal surface area in contact with the intraperitoneal drug $(\mathrm{P}=0.0152)$.

One additional aspect regarding hetastarch as a
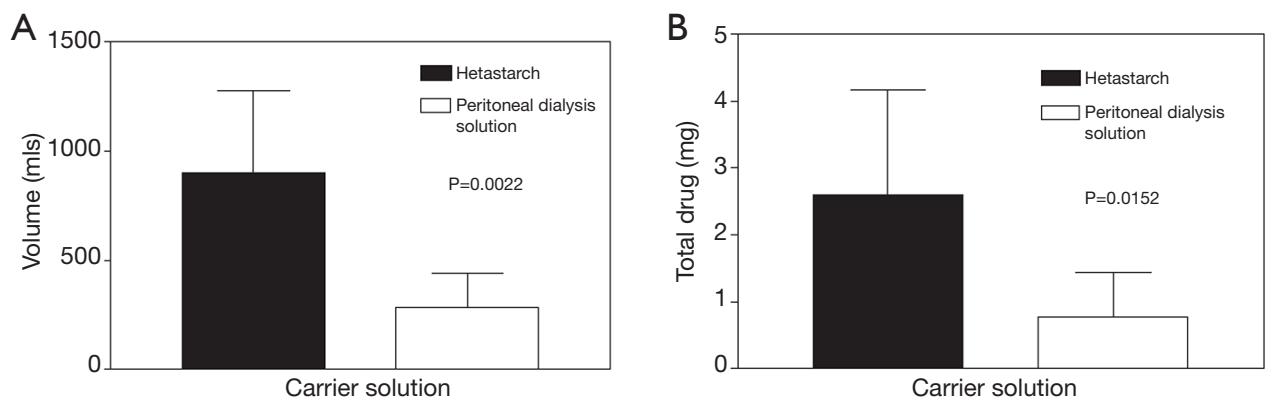

Figure 2 Peritoneal dialysis solution versus hetastarch as a carrier solution for intraperitoneal paclitaxel. (A) Mean volume of fluid remaining in the peritoneal cavity at 23 hours with a starch carrier solution as compared to an aqueous peritoneal dialysis solution. Error bars represent SD. (B) Mean total drug remaining in the peritoneal cavity at 23 hours with hetastarch and peritoneal dialysis solution. Error bars represent SD. Reprinted from (6) with permission. 
carrier solution. It is well known that the intraperitoneal administration of chemotherapy is less likely to be successful as more cycles are given (7). An antiadhesive agent such as a starch solution will separate bowel loops and impede the fibrosis that progressively surrounds the catheter of the intraperitoneal port. Less adverse events with hetastarch as a carrier solution as compared to an aqueous solution may occur in the administration of intraperitoneal chemotherapy.

\section{Penetration of intraperitoneal paclitaxel into tumor nodules}

A major question regarding clinical efficacy of intraperitoneal paclitaxel involves the penetration and then retention of the drug in tumor nodules. Even though there are 2 to $3 \log$ increases in intracavitary drug concentration, increased cancer cell kill has yet to be demonstrated.

Kuh and coworkers pursued in vitro studies to develop regional paclitaxel therapy for localized disease. They used spheroids to determine the barriers to paclitaxel penetration. High tumor cell density was a deterrent to paclitaxel access to the tumor nodules. As apoptosis occurred the cell density barrier to drug penetration was reduced. The factors responsible for improved drug penetration were concentration and time dependent. A reduction of epithelial cells density occurred at 24 hours at high paclitaxel concentration of $120 \mathrm{mM}$. The increased drug penetration was abrupt with marked increase coming in the second 24 hours. In the first 24 hours, paclitaxel penetrated only 15 cell layers as compared to 80 cell layers in the second 24 hours (8). This study shows that prolonged direct contact of a high concentration of the paclitaxel solution with a tumor nodule is necessary to induce apoptosis.

\section{Distribution of intravenous and intraperitoneal paclitaxel}

Soma and coworkers compared in a rabbit model the access of paclitaxel to blood and body organs after intravenous as compared to intraperitoneal administration. Although intravenous paclitaxel showed increased concentration in the blood at time 0 to 1 hour, surprisingly, serum paclitaxel concentrations with intraperitoneal paclitaxel were elevated above intravenous drug from 1 to 24 hours. These data suggest that intraperitoneal paclitaxel is sequestered in the peritoneal space and is slowly transferred through the peritoneal and preperitoneal tissues to the systemic circulation. Measurement of tissue concentrations reinforced this concept of drug distribution with much higher levels of paclitaxel in all organs at $1 / 2$ hour after intravenous administration. However, at 6 hours all organs showed higher paclitaxel concentrations after intraperitoneal administration. This higher paclitaxel concentration was most evident in spleen, ovary, omentum, retroperitoneal lymph nodes and stomach. The intraperitoneal drug persisted in tissues at special risk for peritoneal metastases (9).

\section{Adverse events with intraperitoneal paclitaxel}

In the phase 1 dose escalation study of intraperitoneal paclitaxel, Markman and coworkers noted the marked difference of neutropenia observed with intravenous $v s$. intraperitoneal paclitaxel. Neutropenia is a major dose limiting side effect of paclitaxel administered systemically (10). When intravenous cisplatin and intravenous paclitaxel are combined, great care is taken to prevent severe bone marrow suppression. In contrast, Markman and coworkers observed no neutropenia until the dose of $175 \mathrm{mg} / \mathrm{m}^{2}$ was reached. All 5 patients at this dose level experienced neutropenia. At the dose of $125 \mathrm{mg} / \mathrm{m}^{2}$ there was no neutropenia. The possibility of combining intraperitoneal paclitaxel with intravenous chemotherapy (bidirectional treatment) is a strategy to treat the peritoneal surface as well as the systemic components of ovarian cancer.

In patients given repeated cycles of intraperitoneal chemotherapy, the local toxicity of the drug to bowel surfaces is extremely important. Chemotherapy agents such as doxorubicin, mitomycin $\mathrm{C}$ or melphalan may be safe and effective for a single instillation as with hyperthermic intraperitoneal chemotherapy (HIPEC) but should not be considered for multiple cycles through an intraperitoneal port (11). However, intraperitoneal paclitaxel in a large volume of fluid has no known sclerotic effects and repeated doses by intraperitoneal administration has occurred without adverse outcomes. Intraperitoneal pain was dose related. At $175 \mathrm{mg} / \mathrm{m}^{2}$, two of five patients experienced severe abdominal pain. At the $125 \mathrm{mg} / \mathrm{m}^{2}$ dose, one patient developed abdominal pain at the moderate level. The pain at all dose levels resolved and no episodes of bowel obstruction were reported (1).

However, the well described problems with an intraperitoneal port that are associated with repeated doses of intraperitoneal chemotherapy are also observed 
with intraperitoneal paclitaxel. A major difference in intraperitoneal port-related toxicity is seen when patients are treated prior to or after cytoreductive surgery. Ishigami and coworkers treated 40 patients with peritoneal metastases from gastric cancer. These were unresectable patients. None of these patients experienced abdominal pain. Only one of the 40 patients experienced obstruction of the intraperitoneal catheter (12).

In the ovarian cancer patients reported by Walker et al. in a Gynecologic Oncology Group Study 172, patients had intravenous cisplatin and also intraperitoneal paclitaxel. The incidence of intraperitoneal port-related problems after cytoreductive surgery are evident in approximately half the patients treated (7). These patients were treated after a major cytoreductive surgery for ovarian cancer.

Sugarbaker and Stuart reported on 6 patients treated long-term with intraperitoneal paclitaxel via an intraperitoneal port. All patients had a prior major cytoreductive surgery for malignant peritoneal mesothelioma. Three of those 6 patients had serious portrelated complications. One required port removal because of infection. One had an intense allergic response that was misinterpreted as peritonitis that was treated by laparotomy. A third patient developed a small bowel perforation following the fifth cycle of intraperitoneal paclitaxel. All patients had HIPEC prior to intraperitoneal paclitaxel with approximately 8 weeks between surgery and paclitaxel administered by intraperitoneal port (5). Although the numbers of patients are small, $50 \%$ or patients had a major complication with paclitaxel administered repeatedly through an intraperitoneal port.

These data create a dilemma for the oncologist. In order for intraperitoneal therapy to be maximally effective, the tumor nodule size must be very small. No visible evidence of residual disease is optimal. However, to get to a pharmacologically optimal reduction in tumor load, a major cytoreductive surgery is required. This surgery will inevitably cause fibrous adhesions that work to fix the catheter within the peritoneal space. The fibrous reaction to a fixed catheter proceeds to cause catheter occlusion and limited drug discussion. Methods to reduce adhesions after a major surgical procedure exist but data to suggest an optimal treatment plan are currently not available.

\section{Intraperitoneal paclitaxel for ovarian cancer}

Francis and colleagues with the Gynecologic Oncology Group sought to determine the safe and effective dose of intraperitoneal paclitaxel administered on a weekly basis. Each patient was to receive 16 weekly courses of treatment. Different patients received between $20 \mathrm{mg} / \mathrm{m}^{2} /$ week and $75 \mathrm{mg} / \mathrm{m}^{2} /$ week. With multiple grade II toxicities at $75 \mathrm{mg} / \mathrm{m}^{2} /$ week the recommended dose of intraperitoneal paclitaxel was $60-65 \mathrm{mg} / \mathrm{m}^{2} /$ week. The most common toxicity was abdominal pain. The abdominal pain did not seem to increase as the dose of drug was increased. This suggested that the intraperitoneal instillation of a large volume of fluid was at least, in part, the case of abdominal discomfort or pain. In these patients, myelosuppression was mild except at the highest dose level of treatment. The efficacy of the treatment was difficult to assess because a second-look surgery was not built into the design of the protocol. Most patients had small volume residual disease difficult to measure by CT examination. Twentyfive patients completed at least half of the planned intraperitoneal therapy ( $\geq 8$ weekly doses). Five of these 25 had no clinical evidence of disease progression 8 to 30 months from study entry (13).

This data reported by Francis et al. were incorporated into the intraperitoneal cisplatin and intraperitoneal paclitaxel GOG172 randomized controlled study in ovarian cancer (14). Patients randomized to receive intravenous chemotherapy received both paclitaxel $\left(135 \mathrm{mg} / \mathrm{m}^{2}\right)$ and cisplatin $\left(75 \mathrm{mg} / \mathrm{m}^{2}\right)$. In the intraperitoneal group, paclitaxel was first given intravenously $\left(135 \mathrm{mg} / \mathrm{m}^{2}\right)$. The cisplatin was given at $100 \mathrm{mg} / \mathrm{m}^{2}$ into the peritoneal space. The second intraperitoneal treatment was paclitaxel $\left(60 \mathrm{mg} / \mathrm{m}^{2}\right)$ by intraperitoneal administration on day 8 of the treatment cycle. Treatment was given every 3 weeks for 6 cycles. Survival was improved in the group treated with intraperitoneal cisplatin plus intraperitoneal paclitaxel. Median survival was 49.7 vs. 65.6 months $(\mathrm{P}=0.03)$. This study published in the New England Fournal of Medicine led to the NCI clinical alert encouraging oncologists to use the intraperitoneal route of administration in newly diagnosed ovarian cancer patients following complete cytoreduction. The major criticism of this study is the approximate $50 \%$ incidence of toxicity related to port-based chemotherapy delivery (7). A combined intraperitoneal and intravenous chemotherapy treatment plan recommended for routine use in ovarian cancer patients never was published and led to confusion regarding the use of this treatment strategy.

\section{Neoadjuvant intraperitoneal paclitaxel}

Muñoz-Casares attempted to downstage intraperitoneal 


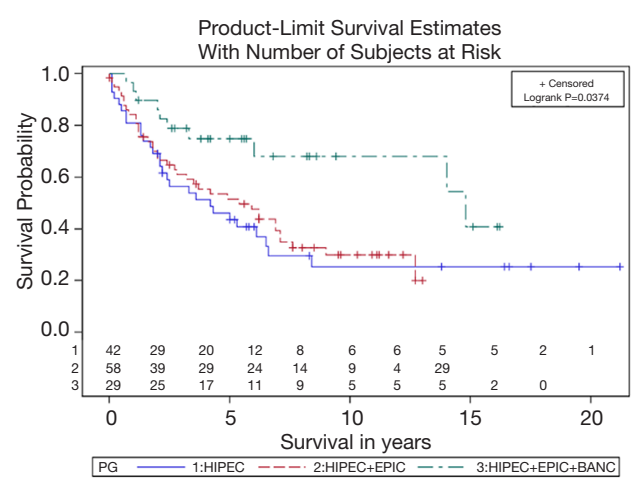

Figure 3 Survival of patients with malignant peritoneal mesothelioma in 3 different treatment groups. Group 1 is HIPEC = hyperthermic perioperative chemotherapy, group 2 is HIPEC $+\mathrm{EPIC}=$ hyperthermic perioperative chemotherapy plus early postoperative intraperitoneal chemotherapy, and group 3 is HIPEC + EPIC + NIPEC $=$ hyperthermic perioperative chemotherapy plus early postoperative intraperitoneal chemotherapy plus normothermic intraperitoneal chemotherapy. Reprinted from (18) with permission.

cancer and thereby improve the surgical cytoreduction of ovarian cancer by using intraperitoneal paclitaxel plus intravenous carboplatin. The paclitaxel was given weekly at $60 \mathrm{mg} / \mathrm{m}^{2} /$ week and the carboplatin repeated every 21 days (AUC 6, for 4 cycles). All patients had laparoscopy prior to their inclusion in this study. The peritoneal cancer index decreased from 21.2 to 14.4 with treatment. The CA125 reduced from 1,053 to 346. All patients who had both paclitaxel and carboplatin as neoadjuvant treatments had a complete cytoreduction to no visible evidence of disease. The 5 -year overall survival in these patients was $62 \%$. The authors conclude that this neoadjuvant bidirectional chemotherapy followed by radical surgery is a safe and effective option for management of primary ovarian cancer (15). Data from a phase III trial is not as yet available.

In a pilot study of 13 patients, de Bree and colleagues used paclitaxel as part of an HIPEC procedure for 2 hours. The dose of paclitaxel was $175 \mathrm{mg} / \mathrm{m}^{2}$. There was no mortality but morbidity was $31 \%$ with 2 patients showing chemotherapy-related neutropenia. Pharmacologic studies were encouraging in that the AUC ratio during the 2 hours of HIPEC was 1,462. Cytotoxic drug concentrations could be detected in peritoneal fluid for a mean of 2.7 days (16). Similarly, Bae and colleagues investigated the use of HIPEC paclitaxel at $175 \mathrm{mg} / \mathrm{m}^{2}$ in 22 patients. The relative risk of disease progression for HIPEC paclitaxel as compared to a control group was favorable $(\mathrm{P}=0.0039)(17)$.

\section{Intraperitoneal paclitaxel in malignant peritoneal mesothelioma}

Sugarbaker and coworkers added a single cycle of paclitaxel used as early postoperative intraperitoneal chemotherapy (EPIC) after patients had received cytoreductive surgery plus HIPEC with cisplatin and doxorubicin. The EPIC paclitaxel was given at $20 \mathrm{mg} / \mathrm{m}^{2}$ five days in a row on postoperative days $1-5$ (total dose $100 \mathrm{mg} / \mathrm{m}^{2}$ ). The 5 -year survival of 42 patients treated with CRS and HIPEC was $44 \%$. For 58 consecutive patients treated with CRS and HIPEC with EPIC paclitaxel, survival was $52 \%$ at 5 years (18). These differences were not significant (Figure 3). Although there was no statistical significance when the group of patients with HIPEC only was compared to the group with HIPEC plus EPIC paclitaxel, there was a significant difference in survival when long-term normothermic intraperitoneal chemotherapy (NIPEC) with pemetrexed and cisplatin were added to HIPEC plus EPIC.

In a pilot study of 6 patients, 6 monthly cycles of normothermic intraperitoneal paclitaxel was added to HIPEC in patients with peritoneal mesothelioma (5). Repeated doses of intraperitoneal paclitaxel seemed to be much more effective than the single cycle of EPIC paclitaxel. The paclitaxel was administered in a similar manner at $20 \mathrm{mg} / \mathrm{m}^{2} /$ day for five days in a row for one week out of every month. There was no systemic chemotherapy given to these patients. Unusually favorable long-term survival was seen in these 6 patients with 4 free of disease at $8,13,18$, and 19 years after definitive cytoreduction of malignant peritoneal mesothelioma. Two patients died at 15 years following treatment. It was noted that 3 of the 6 patients had a grade 3 or 4 adverse event directly related to intraperitoneal chemotherapy administration through an intraperitoneal port.

These two studies performed with the same method for paclitaxel administration seemed to give remarkably different results of treatment when a single cycle of intraperitoneal paclitaxel was compared to multiple cycles of the same drug. No statistically significant improvement in survival was noted with a single cycle of paclitaxel whereas median survival was never reached with 15 -year followup in patients who had multiple cycles of normothermic intraperitoneal paclitaxel through an intraperitoneal port. 


\section{Intraperitoneal paclitaxel in gastric cancer}

Intraperitoneal paclitaxel has been used in an attempt to protect gastric cancer patients at high risk for peritoneal metastases from disease progression on peritoneal surfaces at a later time in the natural history of their disease. Kodera and colleagues in 2011 proposed a randomized study of intraperitoneal paclitaxel versus intravenous paclitaxel in patients with resectable adenocarcinoma of the stomach who had serosal invasion and were at high risk for peritoneal seeding. The dose of intraperitoneal paclitaxel was $60 \mathrm{mg} / \mathrm{m}^{2}$ and it was given on days 1, 15, 22, 29, 43, 50 and 57 following gastrectomy. The control group of patients was to be treated with intravenous paclitaxel at $80 \mathrm{mg} / \mathrm{m}^{2}$ given on the same schedule (19).

Takahashi et al. in 2018 reported results of a phase II multicenter randomized trial that compared intraperitoneal paclitaxel at $60 \mathrm{mg} / \mathrm{m}^{2}$ to intravenous paclitaxel at $80 \mathrm{mg} / \mathrm{m}^{2}$. All patients had gastric cancer at high risk for progression of peritoneal metastases after resection of the primary disease. Patients had linitis plastica, minimal peritoneal metastases, or positive peritoneal cytology. Treatments were on days $0,14,28,42,49$ and 56 postoperatively. Two-year overall survival of intraperitoneal $v s$. intravenous treatments were $64.1 \%$ and $72.3 \%(\mathrm{P}=0.5732)$. Intraperitoneal paclitaxel was not effective to improve survival in gastric cancer patients at high risk for peritoneal recurrence (20).

Imano and colleagues treated 21 patients with gastric cancer with peritoneal metastases or positive cytological findings in peritoneal washings. They performed en bloc D2 lymph node dissection with the gastrectomy. They observed neutropenia in 3 patients (14\%). The dose of the early postoperative intraperitoneal paclitaxel was $80 \mathrm{mg} / \mathrm{m}^{2}$. The chemotherapy solution was dissolved in 1 liter of saline and instilled into the peritoneal cavity at the close of the operation through the closed incision. All drainage tubes into the peritoneal space were clamped for 24 hours after the gastrectomy with paclitaxel EPIC treatment. Pharmacologic studies were performed. The intraperitoneal to plasma area under the concentration curve was 597 to 1. Free intraperitoneal cancer cells could not be recovered following EPIC paclitaxel. One-year survival was $90.5 \%$ and 3 -year survival was $29.8 \%$. The authors suggest that prospective and randomized trials should be used to evaluate this approach to gastric cancer at high risk for peritoneal dissemination (21).

Ishigami and coworkers have performed a randomized phase 3 trial to compare intraperitoneal and intravenous paclitaxel plus S-1 to cisplatin plus S-1 in patients with gastric cancer with peritoneal metastases. The paclitaxel was given $20 \mathrm{mg} / \mathrm{m}^{2}$ into the peritoneal space using an intraperitoneal port. Also, paclitaxel was given at $50 \mathrm{mg} / \mathrm{m}^{2}$ intravenously. Both drugs were given simultaneously at days 1 and 8 plus S-1 $80 \mathrm{mg} / \mathrm{m}^{2} /$ day on days $1-14$ for a 3 -week cycle. Cisplatin was given at $80 \mathrm{mg} / \mathrm{m}^{2} /$ day on days 1 and 21 plus $60 \mathrm{mg} / \mathrm{m}^{2}$ on day 8 of a 5 -week cycle. The primary endpoint of this randomized controlled study was overall survival. Median survival of 164 eligible patients for the intraperitoneal paclitaxel and systemic cisplatin arms were 17.7 and 15.2 months, respectively with a $\mathrm{P}$ value of 0.080 . When the analysis was adjusted for baseline ascites, the hazard ratio was 0.59 (95\% CI, 0.39-0.87; $\mathrm{P}=0.008$ ) in favor of intraperitoneal paclitaxel. The 3 -year overall survival rate was $21.9 \%$ with intraperitoneal paclitaxel and $6 \%$ for systemic cisplatin. Possible clinical benefits for intraperitoneal paclitaxel for gastric cancer with peritoneal seeding was suggested. It should be noted that single drugs (paclitaxel vs. cisplatin) were tested. Bidirectional chemotherapy with cisplatin systemically and paclitaxel intraperitoneally was not tested (22).

The concept of conversion gastrectomy following intravenous and intraperitoneal paclitaxel with oral S-1 was reported by Kitayama and colleagues for patients with advanced gastric cancer and malignant ascites. They studied 64 patients treated as in the prior manuscript by Ishigami with combined intravenous and intraperitoneal paclitaxel. Laparoscopy was used to confirm response in patients who showed apparent shrinkage of their peritoneal nodules as well as negative peritoneal cytology. Thirty-four of the 64 patients went on to receive gastrectomy. Five courses of chemotherapy were the median given prior to conversion surgery. An R0 resection was achieved in $65 \%$ of patients. Median survival and 1-year overall survival was 26.4 months and $82 \%$ on those patients who had the salvage gastrectomy. In patients who did not receive gastrectomy the median survival was 12 months and 1 -year overall survival $26 \%$. The authors conclude that salvage gastrectomy following intravenous and intraperitoneal paclitaxel plus $\mathrm{S}-1$ is a promising treatment for patients with peritoneal dissemination of gastric cancer and malignant ascites (23).

\section{Intraperitoneal paclitaxel in patients with pancreas cancer and malignant ascites}

There is no doubt that the prognosis of patients with pancreas cancer presenting with peritoneal metastases is 


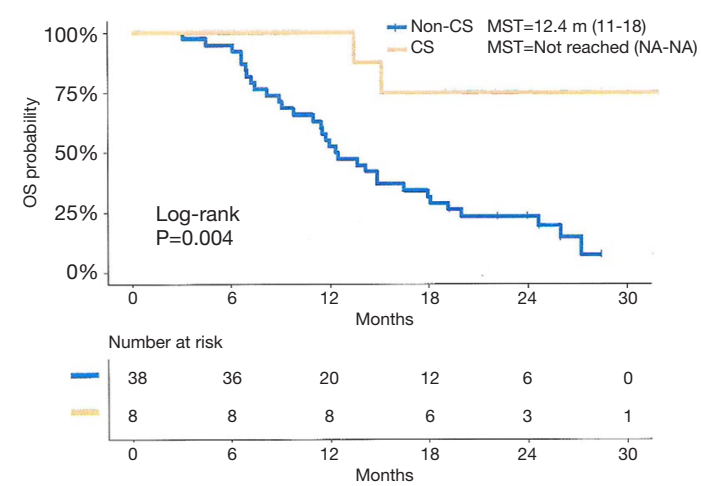

Figure 4 All 36 patients in this study were treated with a combined systemic gemcitabine and nab-paclitaxel. Also, intraperitoneal and intravenous paclitaxel was used. All patients had pancreas cancer with peritoneal dissemination. Eight patients were able, as a result of their neoadjuvant chemotherapy, to undergo a pancreatectomy. The survival of these patients having conversion surgery was statistically significantly prolonged $(\mathrm{P}=0.004)$. Reprinted from (27) with permission.

extremely poor. In a survey of 2,924 Dutch patients, 265 presented with synchronous peritoneal metastases. Their median survival was only 6 weeks (24). Also, Ferrone showed that positive peritoneal cytology in patients with resected pancreas cancer was associated with an 8-month survival as compared to 16 months in the absence positive peritoneal cytology $(\mathrm{P}<0.001)(25)$. In a multicenter study of intravenous and intraperitoneal paclitaxel with S-1, Satoi and colleagues reported on 33 patients who had peritoneal dissemination without other organ metastases from pancreas cancer (26). Seven patients had pancreas head cancer and 23 patients had pancreas body/tail disease. With the combined intravenous and intraperitoneal paclitaxel plus $\mathrm{S}-1$, the median survival was 16.3 months with a 1 -year survival of $62 \%$. Eight patients underwent conversion surgery with a significantly higher survival than the non-surgical patients $(\mathrm{P}=0.0062)$.

A bidirectional chemotherapy regimen for pancreas ductal adenocarcinoma with peritoneal metastases was reported by Yamada and colleagues (27). In these poor prognosis patients, they used systemic chemotherapy with nab-paclitaxel and gemcitabine in an attempt to control systemic disease. A combination of intravenous and intraperitoneal paclitaxel with S-1 was used in an attempt to control the peritoneal surface component of the pancreas adenocarcinoma. Fifty patients were enrolled in this phase I/II study. In these protocol patients, the peritoneal metastases were present; however, the disease was otherwise resectable. The intraperitoneal treatments were given through an intraperitoneal port. Gemcitabine and nabpaclitaxel were given on days 1,8 and 15 followed by 1 week of rest. The intraperitoneal and systemic paclitaxel was repeated every 4 weeks. Response according to RECIST was $48.8 \%$ and disease control $95.3 \%$. Peritoneal washings turned negative with this bidirectional treatment in $39 \%$ of patients along with a disappearance of the malignant ascites. In 8 patients, a conversion surgery was performed (17.4\%) and 7 of these 8 patients had an R0 resection. The patients who underwent conversion surgery survived significantly longer than those who did not undergo conversion surgery with a median survival not reached versus 12.4 months $(\mathrm{P}=0.0040)$. This combination of systemic and localregional (intraperitoneal) treatments produced excellent palliation with prolonged survival and allowed a conversion surgery which showed prolonged survival in a subset of pancreas cancer patients with an extremely poor prognosis (Figure 4).

\section{Summary}

The unique pharmacokinetic properties of intraperitoneal paclitaxel make it a candidate for testing in a large number of gastrointestinal and gynecologic malignancies. Data would strongly suggest that systemic response to paclitaxel may have little to do with the local-regional control that this drug can achieve through repeated intraperitoneal administration. A responsive malignancy and an unusually intense exposure to chemotherapy given through the intraperitoneal route of administration would be the ideal clinical situation. Of course, ovarian cancer is the first disease in this category that comes to mind. However, other responsive diseases would be endometrial cancer with peritoneal metastases and gastric cancer being treated for peritoneal dissemination. Because of the problems with intraperitoneal ports and drug access to peritoneal nodules, treatment protocols prior to cytoreductive surgery are preferred. This neoadjuvant approach is almost always successful in palliating the malignant ascites. However, its efficacy in treating gross disease is less predictable. Single cycles of intraperitoneal paclitaxel are minimally effective. Repeated doses of regional chemotherapy are necessary in order to achieve a significant benefit. The concept of bidirectional chemotherapy needs further exploration. The best chemotherapy agents for systemic disease should be given systemically. Usually, cisplatin is a part of this regimen 
and its neurologic and marrow suppression effects need to be carefully monitored. Large doses of intraperitoneal paclitaxel up to $120 \mathrm{mg}$ on a monthly basis or $60 \mathrm{mg}$ on a weekly basis can be combined with these systemic agents with minimal or no additional hematologic toxicity. These bidirectional protocols may lead to conversion surgery which has been shown to markedly prolong the survival of groups of patients whose longevity in the past was limited to weeks.

\section{Acknowledgments}

Funding: None.

\section{Footnote}

Provenance and Peer Review: This article was commissioned by the editorial office, Journal of Gastrointestinal Oncology, for the focused issue "Intraperitoneal Chemotherapy for Peritoneal Metastases: HIPEC, EPIC, NIPEC, PIPAC and More". This article has undergone external peer review.

Conflicts of Interest: The author has completed the ICMJE uniform disclosure form (available at http://dx. doi. org/10.21037/jgo-2020-03). The focused issue was sponsored by the Peritoneal Surface Oncology Group International (PSOGI). PHS served as the unpaid Guest Editor of the focused issue. The author has no other conflicts of interest to declare.

Ethical Statement: The author is accountable for all aspects of the work in ensuring that questions related to the accuracy or integrity of any part of the work are appropriately investigated and resolved.

Open Access Statement: This is an Open Access article distributed in accordance with the Creative Commons Attribution-NonCommercial-NoDerivs 4.0 International License (CC BY-NC-ND 4.0), which permits the noncommercial replication and distribution of the article with the strict proviso that no changes or edits are made and the original work is properly cited (including links to both the formal publication through the relevant DOI and the license). See: https://creativecommons.org/licenses/by-nc-nd/4.0/.

\section{References}

1. Markman M, Rowinsky E, Hakes T, et al. Phase I trial of intraperitoneal taxol: A Gynecologic Oncology Group study. J Clin Oncol 1992;10:1485-91.

2. Rowinsky EK, Cazenave LA, Donehower RC. Taxol: A novel investigational antimicrotubule agent. J Natl Cancer Inst 1990;82:1247-59.

3. Markman M, Bundy BN, Alberts DS, et al. Phase III trial of standard-dose intravenous cisplatin plus paclitaxel versus moderately high-dose carboplatin followed by intravenous paclitaxel and intraperitoneal cisplatin in small-volume stage III ovarian carcinoma: An intergroup study of the Gynecologic Oncology Group, Southwest Oncology Group, and Eastern Cooperative Oncology Group. J Clin Oncol 2001;19:1001-7.

4. Mohamed F, Sugarbaker PH. Intraperitoneal taxanes. Surg Oncol Clin N Am 2003;12:825-33.

5. Sugarbaker PH, Stuart OA. Unusually favorable outcome of 6 consecutive patients with diffuse malignant peritoneal mesothelioma treated with intraperitoneal paclitaxel. Surg Oncol 2020;33:96-9.

6. Mohamed F, Marchettini P, Stuart OA, et al. A comparison of hetastarch and peritoneal dialysis solution for intraperitoneal chemotherapy delivery. Eur J Surg Oncol 2003;29:261-5.

7. Walker JL, Huang $\mathrm{H}$, Armstrong D, et al. Intraperitoneal catheter outcomes in a phase III trial of intravenous versus intraperitoneal chemotherapy in optimal stage III ovarian and primary peritoneal cancer: A Gynecologic Oncology Group study. Gynecol Oncol 2006;100:27-32.

8. Kuh HJ, Jang SH, Wientjes MG, et al. Determinants of paclitaxel penetration and accumulation in human solid tumor. J Pharmacol Exp Ther 1999;290:871-80.

9. Soma D, Kitayama J, Ishigami H, et al. Different tissue distribution of paclitaxel with intravenous and intraperitoneal administration. J Surg Res 2009;155:142-6.

10. Einzig AI, Wiernik PH, Sasloff J, et al. Phase II study and long-term follow-up of patients treated with taxol for advanced ovarian adenocarcinoma. J Clin Oncol 1992;10:1748-53.

11. Sugarbaker PH. Intraperitoneal delivery of chemotherapeutic agents for the treatment of peritoneal metastases: Current challenges and how to overcome them. Expert Opin Drug Deliv 2019;16:1393-401.

12. Ishigami H, Kitayama J, Kaisaki S, et al. Phase II study of weekly intravenous and intraperitoneal paclitaxel combined with S-1 for advanced gastric cancer with peritoneal metastases. Ann Oncol 2010;21:67-70.

13. Francis P, Rowinsky E, Schneider J, et al. Phase I feasibility and pharmacologic study of weekly intraperitoneal 
paclitaxel: A Gynecologic Oncology Group pilot study. J Clin Oncol 1995;13:2961-7.

14. Armstrong DK, Bundy B, Wenzel L, et al. Intraperitoneal cisplatin and paclitaxel in ovarian cancer. $\mathrm{N}$ Engl J Med 2006;354:34-43.

15. Muñoz-Casares FC, Rufián S, Arjona-Sánchez Á, et al. Neoadjuvant intraperitoneal chemotherapy with paclitaxel for the radical surgical treatment of peritoneal carcinomatosis in ovarian cancer: a prospective pilot study. Cancer Chemother Pharmacol 2011;68:267-74.

16. de Bree E, Rosing H, Filis D, et al. Cytoreductive surgery and intraoperative hyperthermic intraperitoneal chemotherapy with paclitaxel: a clinical and pharmacokinetic study. Ann Surg Oncol 2008;15:1183-92.

17. Bae JH, Lee JM, Ryu KS, et al. Treatment of ovarian cancer with paclitaxel- or carboplatin-based intraperitoneal hyperthermic chemotherapy during secondary surgery. Gynecol Oncol 2007;106:193-200.

18. Sugarbaker PH, Chang D. Long-term regional chemotherapy for patients with epithelial malignant peritoneal mesothelioma results in improved survival. Eur J Surg Oncol 2017;43:1228-35.

19. Kodera $Y$, Imano M, Yoshikawa T, et al. A randomized phase II trial to test the efficacy of intra-peritoneal paclitaxel for gastric cancer with high risk for the peritoneal metastases (INPACT Trial). Jpn J Clin Oncol 2011;41:283-6.

20. Takahashi N, Kanda M, Yoshikawa T, et al. A randomized phase II multicenter trial to explore efficacy of weekly intraperitoneal in comparison with intravenous paclitaxel administered immediately after gastrectomy to the patients with high risk of peritoneal recurrence: final results of the

Cite this article as: Sugarbaker PH. Intraperitoneal paclitaxel: pharmacology, clinical results and future prospects. J Gastrointest Oncol 2021;12(Suppl 1):S231-S239. doi: 10.21037/ jgo-2020-03
INPACT trial. Gastric Cancer 2018;21:1014-23.

21. Imano $M$, Inamoto $H$, Itoh $T$, et al. Safety of intraperitoneal administration of paclitaxel after gastrectomy with en-bloc D2 lymph node dissection. J Surg Oncol 2012;105:43-7.

22. Ishigami H, Fujiwara Y, Fukushima R, et al. Phase III trial comparing intraperitoneal and intravenous paclitaxel plus S-1 versus cisplatin plus S-1 in patients with gastric cancer with peritoneal metastases: PHOENIX-GC trial. J Clin Oncol 2018;36:1922-9.

23. Kitayama J, Ishigami H, Yamaguchi H, et al. Salvage gastrectomy after intravenous and intraperitoneal paclitaxel (PTX) administration with oral S-1 for peritoneal dissemination of advanced gastric cancer with malignant ascites. Ann Surg Oncol 2014;21:539-46.

24. Thomassen I, Lemmens V, Nienhuijs SW, et al. Incidence, prognosis, and possible treatment strategies of peritoneal carcinomatosis of pancreatic origin. A population-based study. Pancreas 2013;42:72-5.

25. Ferrone CR, Haas B, Tang L, et al. The influence of positive peritoneal cytology on survival in patients with pancreatic adenocarcinoma. J Gastrointest Surg 2006;10:1347-53.

26. Satoi S, Fujii T, Yanagimoto H, et al. Multicenter phase II study of intravenous and intraperitoneal paclitaxel with S-1 for pancreatic ductal adenocarcinoma patients with peritoneal metastases. Ann Surg 2017;265:397-401.

27. Yamada S, Fujii T, Yamamoto T, et al. Multicenter phase I/II study of intravenous gemcitabine + nab-paclitaxel combined with intraperitoneal paclitaxel for pancreatic ductal adenocarcinoma patients with peritoneal metastasis. J Clin Oncol 2020;38:702. 